Phase sensitivity of high harmonic transient grating spectroscopy

This article has been downloaded from IOPscience. Please scroll down to see the full text article.

2010 J. Phys. B: At. Mol. Opt. Phys. 43065401

(http://iopscience.iop.org/0953-4075/43/6/065401)

The Table of Contents and more related content is available

Download details:

IP Address: 132.246.118.152

The article was downloaded on 03/03/2010 at 13:13

Please note that terms and conditions apply. 


\title{
Phase sensitivity of high harmonic transient grating spectroscopy
}

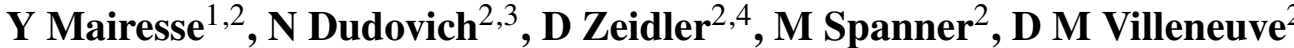 \\ and P B Corkum ${ }^{2,5}$ \\ ${ }^{1}$ CELIA, Université Bordeaux 1, UMR5107 (CNRS, Bordeaux 1, CEA), 351, cours de la libération, \\ 33405 Talence, France \\ ${ }^{2}$ National Research Council of Canada, 100 Sussex Drive, Ottawa, Ontario K1A 0R6, Canada \\ ${ }^{3}$ Department of Physics of Complex Systems, Weizmann Institute of Science, Rehovot 76100, Israel \\ ${ }^{4}$ Carl Zeiss SMT AG, D-73447 Oberkochen, Germany \\ ${ }^{5}$ University of Ottawa, Ottawa, ON, Canada
}

Received 30 October 2009, in final form 12 January 2010

Published 2 March 2010

Online at stacks.iop.org/JPhysB/43/065401

\begin{abstract}
We study the spatial profile of high order harmonics generated by a transient grating of rotational excitation. We show that the phase modulation of the harmonic emission as a function of molecular alignment is encoded in the diffraction pattern. In molecular nitrogen, the phase difference between aligned and isotropic molecules decreases from $1.6 \mathrm{rad}$ for harmonic 19 to less than 0.3 rad for harmonic 27. In $\mathrm{CO}_{2}$ we observe a strong phase jump for the highest harmonics. The position of this phase jump in the harmonic spectrum depends on the laser intensity, reflecting the contribution from multiple molecular orbitals to the harmonic emission.
\end{abstract}

(Some figures in this article are in colour only in the electronic version)

\section{Introduction}

The high order harmonics which are emitted when a molecule is submitted to a strong laser field can be used to retrieve structural information on the generating medium [1]. The perspective of using the high harmonic emission to image molecular dynamics has stimulated a lot of interest and triggered many experiments [2]. The structural information is not only encoded in the harmonic amplitudes but also in their polarization [3] and phase [4-8]. In this paper, we use a new approach based on transient grating spectroscopy to measure this phase.

Transient grating methods transfer amplitude and phase information about the light-matter interaction from the temporal domain, where it sits on a large background, to the spatial domain where it is observed against a zero background [9]. These two properties, phase sensitivity and background suppression, make transient gratings an important tool in conventional ultrafast measurements. Recently, we have shown that this technique could be transfered to the extreme nonlinear regime [10]. By studying the spatial profile of a high harmonic generated in rotationally excited nitrogen molecules we have demonstrated a dramatic increase in the constrast of the detection of revivals of molecular alignment. Here we take this technique one step further. We study the diffraction pattern as a function of harmonic order and generating gas. We show that the harmonic-phase modulation as a function of molecular alignment can be retrieved from the diffraction pattern. Very different behaviours are observed in $\mathrm{N}_{2}$ and $\mathrm{CO}_{2}$, demonstrating the sensitivity of the technique to the molecular structure.

\section{Building a sinusoidal grating of molecular alignment}

In this section, we present our experimental setup and perform a theoretical study of the molecular alignment grating to determine proper experimental conditions.

The experimental setup is shown in figure 1 and was described in [10]. We use a 50-Hz 30-fs 800-nm Ti:Sa laser system. Two horizontally polarized crossed pump beams are focused with a $50 \mathrm{~cm}$ lens in a pulsed gas jet where their optical interference produces a spatially modulated rotational excitation pattern. The pump beam waist is typically $\sim 150 \mu \mathrm{m}$ and the horizontal fringes are separated by $13 \mu \mathrm{m}$. 


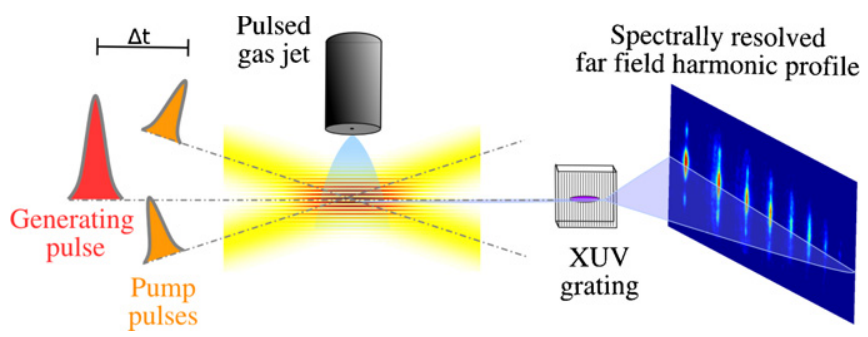

Figure 1. Experimental setup. A grating of molecular excitation is created by the two synchronized crossed laser pulses. The delayed central pulse generates high harmonics in the excited medium. The harmonics are spectrally resolved in the horizontal plane by an XUV spectrometer, and spatially resolved in the vertical plane.

In the regions of maximum pump intensity, a rotational wavepacket is efficiently created $[11,12]$. By contrast, in the planes of zero pump intensity, the alignment distribution remains isotropic. We use an intense delayed probe pulse to generate high harmonics in the structured medium. The probe pulse is horizontally polarized and its beam waist is $100 \mu \mathrm{m}$. The spatial modulation of the generating medium results in the emission of diffracted beams of high harmonic radiation.

The molecular alignment grating can be considered as a transient diffractive mask, which induces both amplitude and phase modulations of the emission along the vertical dimension $x$ in the generating medium. The harmonic field $E_{q}$ at the exit of the generating medium can then be written as $E_{q}(x)=E_{q}^{0}(x) T_{q}(x) \mathrm{e}^{\mathrm{i} \delta \phi_{q}(x)}$, where $E_{q}^{0}$ is the unperturbed harmonic field, and $T_{q}$ and $\delta \phi_{q}$ are the amplitude and phase modulations induced by the grating [13]. We measure the far-field harmonic profile, which is the Fourier transform of $E_{q}(x)$.

The aim of our experiment is to extract the harmonic amplitude and phase modulations from the diffraction patterns. The exact shape of the grating is a crucial parameter in such a measurement and we would like to use sinusoidal gratings in order to keep the analysis straightforward. However, using a sinusoidal modulation of the pump intensity does not ensure that $T_{q}(x)$ and $\delta \phi_{q}(x)$ will be sinusoidal: first, the rotational excitation process is very nonlinear, so that the molecular alignment grating could be inharmonic; second, if the harmonic amplitude and phase are nonlinear functions of the degree of molecular alignment, then $T_{q}(x)$ and $\delta \phi_{q}(x)$ will also be inharmonic.

In order to determine appropriate experimental conditions, we first perform a theoretical study of the profile of the alignment grating in nitrogen molecules. To evaluate this profile, we need to calculate the evolution of the degree of alignment as a function of the pump intensity. We have calculated the rotational wavepacket dynamics with the method detailed in [14] for a rotational temperature of $30 \mathrm{~K}$ and for different pump intensities. We extract the degree of molecular alignment $\left\langle\cos ^{2} \theta\right\rangle$ at $\Delta t=4.1$ ps (figure 2(a)). For isotropic molecules $\left(I^{\text {pump }}=0\right)\left\langle\cos ^{2} \theta\right\rangle=0.33$. For pump intensities below $5 \times 10^{13} \mathrm{~W} \mathrm{~cm}^{-2},\left\langle\cos ^{2} \theta\right\rangle$ increases quasi-linearly with intensity. As the intensity further increases, $\left\langle\cos ^{2} \theta\right\rangle$ saturates to a maximum value 0.65 at $I^{\text {pump }}=$ $9 \times 10^{13} \mathrm{~W} \mathrm{~cm}^{-2}$ and then decreases. This behaviour appears paradoxical: the degree of molecular alignment is expected to reach higher values as the pump intensity increases. However what is plotted in figure 2(a) is the value of $\left\langle\cos ^{2} \theta\right\rangle$ at a fixed delay, $\Delta t=4.1 \mathrm{ps}$. As the pump intensity increases, the bandwidth of the rotational wavepacket increases, so that the revival structures become sharper. The delay at which the degree of molecular alignment is maximum increases. Consequently, the value of $\left\langle\cos ^{2} \theta\right\rangle$ at 4.1 ps decreases.

For low enough pump intensities $\left(I^{\text {pump }}=5 \times 10^{13} \mathrm{~W}\right.$ $\left.\mathrm{cm}^{-2}\right),\left\langle\cos ^{2} \theta\right\rangle$ is linear and the alignment grating has a sinusoidal profile similar to that of pump intensity grating (figure 2(b)). An additional condition is required to ensure that the diffractive mask is sinusoidal: the harmonic intensity and phase should vary linearly with $\left\langle\cos ^{2} \theta\right\rangle$. This can be checked by measuring the diffraction pattern obtained: a pure harmonic grating will give only first-order diffraction peaks, while an inharmonic grating will produce higher orders.

Figure 2(d) shows the measured spatial profiles of harmonic 17 corresponding to two cases: $I^{\text {pump }}=5 \times 10^{13} \mathrm{~W}$ $\mathrm{cm}^{-2}$ and $I^{\text {pump }}=2 \times 10^{14} \mathrm{~W} \mathrm{~cm}^{-2}$. At high intensity (black line), there are strong first- and second-order diffraction peaks. The asymmetry between positive and negative diffraction order is due to a slight misalignment of the probe beam axis with respect to the grating normal. The presence of second-order peaks is a signature of the distortions of the alignment grating [10]. When the pump intensity is lowered to $I^{\text {pump }}=5 \times 10^{13} \mathrm{~W} \mathrm{~cm}^{-2}$, only first-order peaks are obtained. This shows that in this case, the modulation of the harmonic emission is sinusoidal like the alignment grating (figure 2(b)). One can conclude from these observations that the harmonic intensity modulation $T_{q}(x)$ and phase modulation $\delta \phi_{q}(x)$ are proportional to the degree of molecular alignment. In the following we will stay in this low pump intensity domain $\left(I^{\text {pump }}=5 \times 10^{13} \mathrm{~W} \mathrm{~cm}^{-2}\right)$ in order to stay in the linear regime and ensure straightforward retrieval of the phase. We can then write

$$
T_{q}(x)=C_{q}+\left(C_{q}-1\right) \cos (2 \pi x / \Lambda)
$$

and

$$
\delta \phi_{q}(x)=\frac{1}{2} \delta \phi_{q}^{0} \cos (2 \pi x / \Lambda),
$$

where $C_{q}$ is characteristic of the contrast of the amplitude grating, $\Lambda$ is the spatial period of the grating and $\delta \phi_{q}^{0}$ is the phase modulation depth.

\section{Diffraction efficiency}

The sensitivity of harmonic emission to molecular alignment depends on the harmonic order. In this section, we study the diffraction pattern for different harmonics and extract the diffraction efficiency, which will be used to measure the phase modulation.

Figure 3(a) shows a spectrally resolved far-field harmonic profile obtained by generating high harmonics in isotropic $\mathrm{N}_{2}$ molecules at $\sim 1.5 \times 10^{14} \mathrm{~W} \mathrm{~cm}^{-2}$. The harmonics are constituted of a bright central lobe surrounded by a weaker more divergent part. These two regions of the spatial profile correspond respectively to short and long electron trajectories 

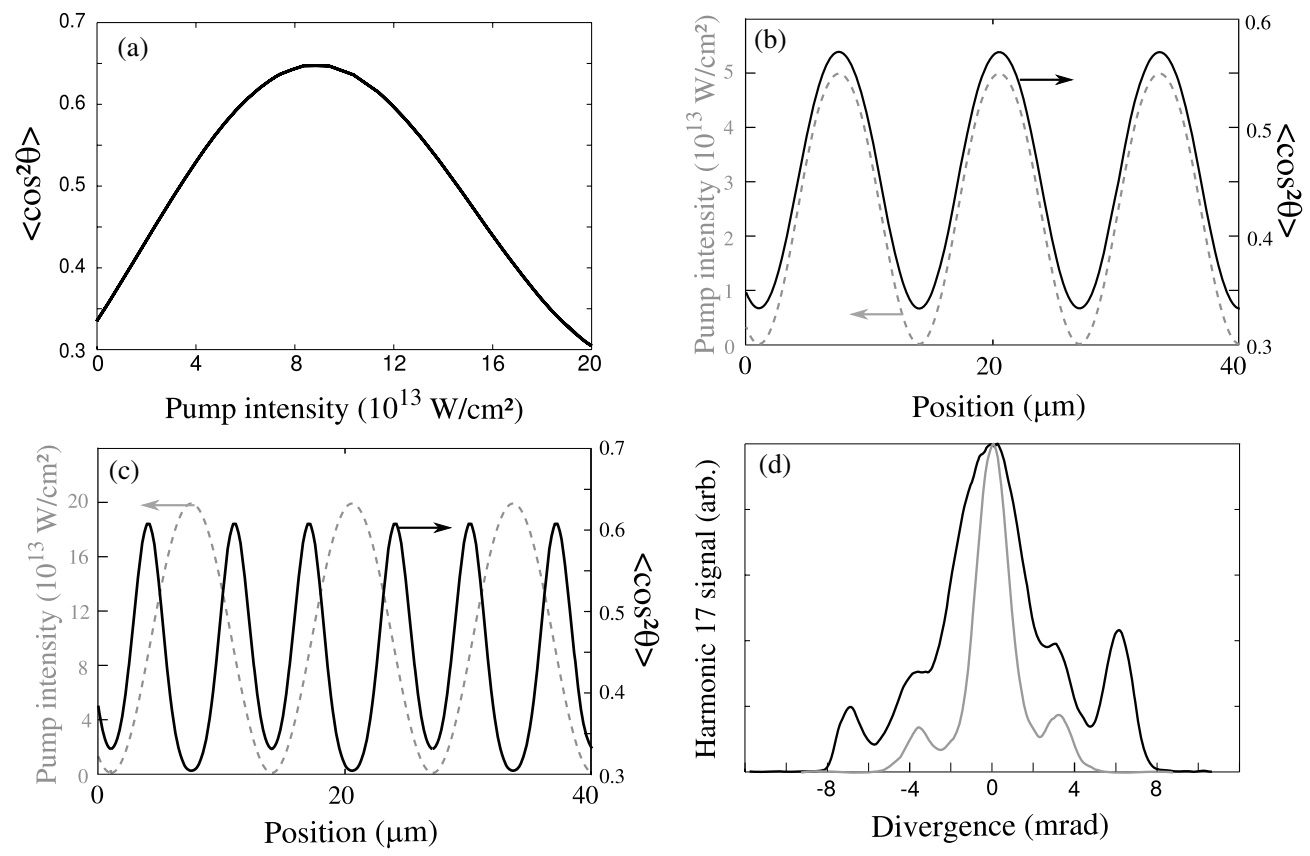

Figure 2. (a) Calculated degree of molecular alignment in nitrogen molecules at $\Delta t=4.1 \mathrm{ps}$ as a function of pump laser intensity. (b-c) Pump intensity beam profile (grey) and resulting molecular alignment grating (black) at $I^{\text {pump }}=5 \times 10^{13} \mathrm{~W} \mathrm{~cm}^{-2}(\mathrm{~b})$ and $2 \times 10^{14} \mathrm{~W} \mathrm{~cm}{ }^{-2}$ (c). (d) Measured spatial profiles of harmonic 17 generated in $\mathrm{N}_{2}$ at $\Delta t=4.1 \mathrm{ps}$, with $I^{\text {pump }}=2 \times 10^{14} \mathrm{~W} \mathrm{~cm}^{-2}$ (black) and $5 \times 10^{13} \mathrm{~W}$ $\mathrm{cm}^{-2}$ (grey).

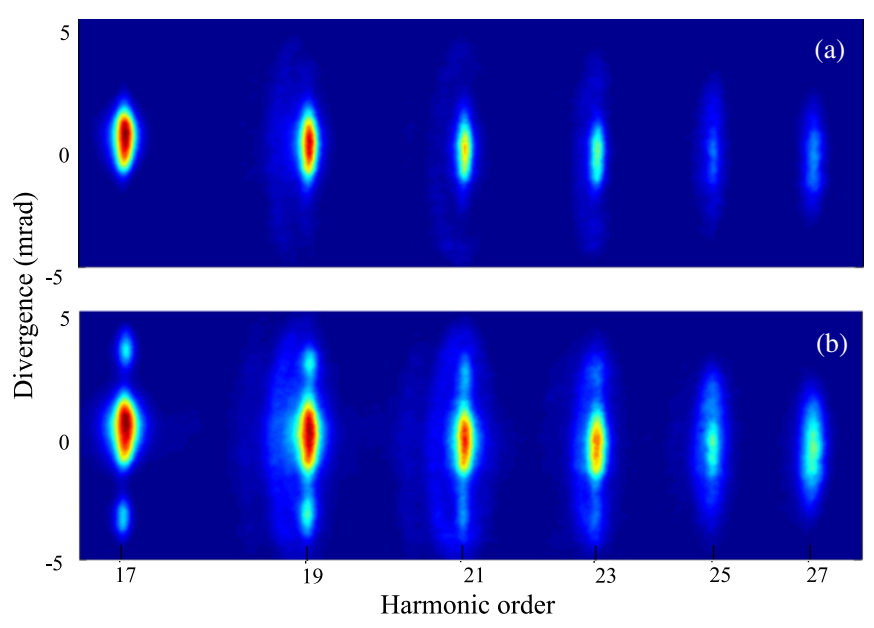

Figure 3. Spectrally resolved far-field harmonic profiles obtained (a) at random alignment in $\mathrm{N}_{2}$ at $1.5 \times 10^{14} \mathrm{~W} \mathrm{~cm}^{-2}$ and (b) at the pump-probe delay that maximizes the alignment $(\Delta t=4.1 \mathrm{ps})$ with $I^{\text {pump }}=5 \times 10^{13} \mathrm{~W} \mathrm{~cm}^{-2}$.

in the generating mechanism [15] (for harmonic 17 the long trajectories are below the detection threshold). When the grating of molecular alignment is applied $(\Delta t=4.1 \mathrm{ps}$ with $\left.I^{\text {pump }}=5 \times 10^{13} \mathrm{~W} \mathrm{~cm}^{-2}\right)$, first-order diffraction peaks appear around the central lobe of the lowest harmonics (figure 3(b)).

The diffraction efficiency can be defined as the fraction of the signal that appears in a single diffraction order. To evaluate it, we calculate the difference between the normalized spatial profiles obtained with (figure 3(b)) and without (figure 3(a)) the alignment grating. The diffraction efficiency is half the integral of this difference. This calculation is valid as long as the diffracted peaks are well separated from the central lobe. The strongest diffraction efficiency is obtained for H19 (12.3\%). As the harmonic order increases, the central lobe becomes broader and the diffraction peaks are closer to the axis. This overlap between central lobe and diffraction peaks introduces an uncertainty on the diffraction efficiency. Increasing the angle between the two pump beams would result in a better separation of the diffraction peaks and a higher accuracy on the measurement. For the two highest harmonics (H25 and H27), we observe no clear diffraction peaks. The spatial profile is slightly broader with the alignment grating and our analysis gives a very low diffraction efficiency $(2.5 \pm 1 \%)$.

\section{Amplitude and phase}

The diffraction of the harmonic light results from both amplitude and phase modulations of the emission in the generating medium. In order to disentangle amplitude and phase we need to evaluate their relative contributions to the diffraction efficiency.

First, we need to determine the contrast of the amplitude modulation $T_{q}(x)$. To that purpose we compare the total signal, integrated over the diffraction pattern, obtained with and without the alignment grating. For harmonic 17, we find that the presence of the alignment grating increases this signal by a factor 2 . The square root of this signal increase is equal to the average value of the grating transmission $T_{q}(x)$ : $\left\langle T_{q}\right\rangle=C_{q}$. Thus, the comparison of the total signal with and without the grating enables us to determine $C_{q}$ for each harmonic, i.e. the contrast of the amplitude modualtion.

In a second step, we simulate the diffraction efficiency of a grating consisting of the measured amplitude modulation and of a sinusoidal phase modulation $\delta \phi_{q}(x)$ of variable depth $\delta \phi_{q}^{0}$. 


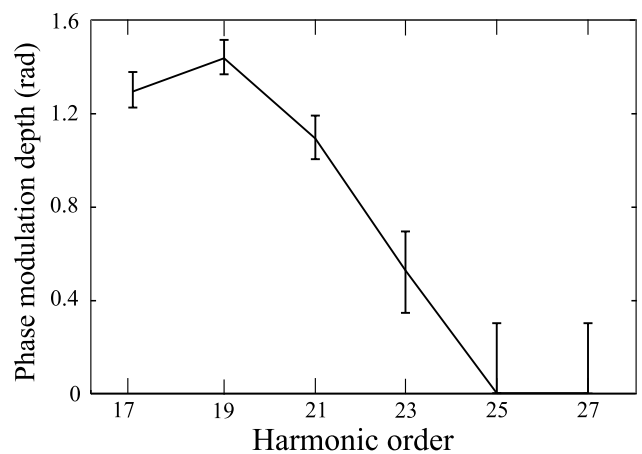

Figure 4. Retrieved modulation depth of the phase grating in $\mathrm{N}_{2}$ at $\Delta t=4.1$ ps with $I^{\text {pump }}=5 \times 10^{13} \mathrm{~W} \mathrm{~cm}^{-2}$.

For $q=17$, we find that the first-order diffraction efficiency increases from $0.7 \%$ to $33 \%$ as $\delta \phi_{q}^{0}$ increases from 0 to $3.7 \mathrm{rad}$. Stronger phase modulations result in a depletion of the firstorder diffraction peak and appearance of higher order peaks.

Last, we use the measured diffraction efficiency to map the corresponding phase modulation depth. For H17, the measured diffraction efficiency $(10.5 \%)$ corresponds to $\delta \phi_{17}^{0}=1.28 \mathrm{rad}$. The diffraction efficiency varies linearly with the phase around this value and thus constitutes a good measurement of the phase modulation.

We repeat this procedure for each harmonic and obtain the evolution of the phase modualtion depth as a function of harmonic order shown in figure 4. Our measurements show that the phase modulation decreases as the harmonic order increases. In particular, we observe no significant phase modulation around $\mathrm{H} 25$, where a spectral minimum was reported [1] and attributed to two-centre interference in the generation process [16]. This is consistent with the fact that this spectral minimum was found to be independent of molecular alignment angle [17]. Our measurements are also consistent with recent studies of the harmonic emission times in $\mathrm{N}_{2}$, which revealed a phase jump as a function of the harmonic order even in randomly aligned molecules [18] and weaker variations as a function of molecular alignment [19].

\section{Measurements in $\mathrm{CO}_{2}$}

We have performed similar measurements in $\mathrm{CO}_{2}$ with $I^{\text {pump }}=5 \times 10^{13} \mathrm{~W} \mathrm{~cm}^{-2}$. The spatial profiles obtained when the pump-probe delay is $\Delta t=20.2 \mathrm{ps}$ are shown in figure 5(a). This delay is far from the half revival period of $\mathrm{CO}_{2}\left(T_{r} / 2=21.4 \mathrm{ps}\right)$. However, even away from revivals the molecular alignment distribution is slightly affected by the pump pulses: there is a permanent 'incoherent' alignment [14]. This creates a permanent grating in the medium [10]. The effect of this grating can be seen in the spatial profile of the highest harmonics (above H23), which show some diffraction. As the delay is set to maximize molecular alignment $(\Delta t=21.2 \mathrm{ps})$ diffraction peaks appear on the sides of the lowest harmonics, while the highest harmonics show a depletion of the on-axis signal (figure 5(b)).

Strong phase gratings can indeed induce a depletion of the zero order. In addition, strong phase modulations of the

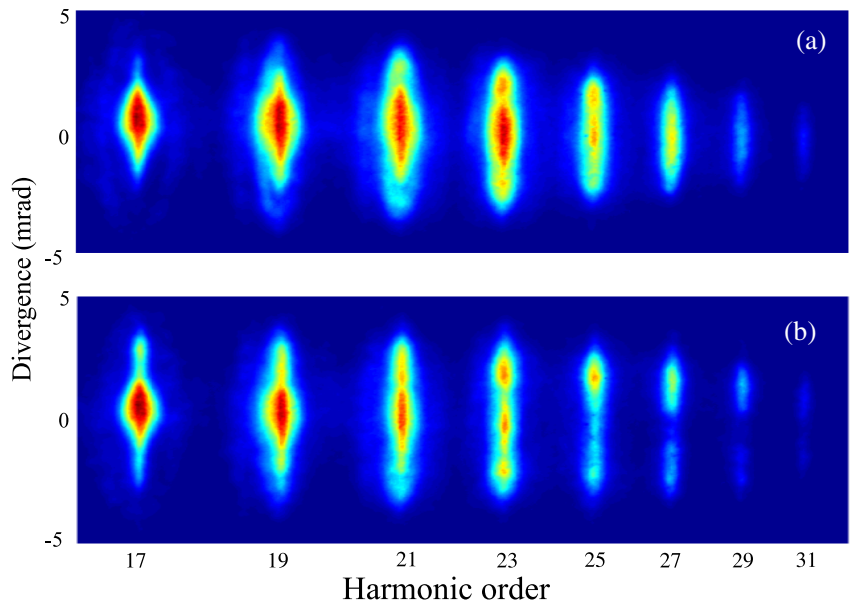

Figure 5. Spectrally resolved far-field harmonic profiles obtained in $\mathrm{CO}_{2}$ at $1.5 \times 10^{14} \mathrm{~W} \mathrm{~cm}^{-2}$ with $I^{\text {pump }}=5 \times 10^{14} \mathrm{~W} \mathrm{~cm}^{-2}$, (a) at $\Delta t=20 \mathrm{ps}$ and (b) $\Delta t=21.2 \mathrm{ps}$.

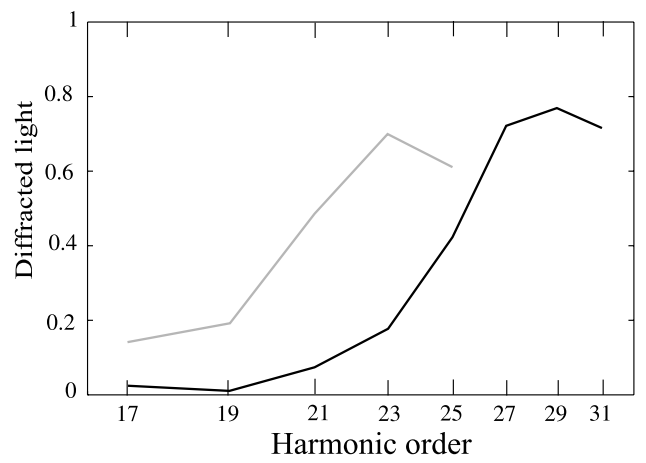

Figure 6. Normalized diffracted signal as a function of harmonic order at $1.5 \times 10^{14} \mathrm{~W} \mathrm{~cm}^{-2}$ (black) and $1 \times 10^{14} \mathrm{~W} \mathrm{~cm}^{-2}$ (grey) generating intensity.

harmonic emission in the near field is likely to result in a loss of signal due to poor phase matching [13]. Thus, while we can affirm that the depletion of the on-axis signal and the appearance of side lobes is associated with a strong phase modulation, it is difficult to extract the exact value of this phase. From our simulations on diffraction and phase matching [13], we estimate that the phase modulation depth is higher than $2 \mathrm{rad}$.

Rather than extracting phase modulation depth, we evaluate a normalized diffracted signal. We define $I_{\mathrm{on}}$ as the ratio of the on-axis harmonic signal (at the centre of the beam) and the total harmonic signal. We normalize it to the case where the delay is off the revival. We define the normalized diffracted signal as the off-axis signal $I_{\text {off }}=1-I_{\mathrm{on}}$ to obtain the curve plotted in figure $6 . I_{\text {off }}$ is 0 when there is no diffraction and increases as the diffraction efficiency increases.

The evolution of the diffracted signal with harmonic order is shown in figure 6. It confirms that the highest harmonics are strongly diffracted, i.e. that they experience a strong phase modulation. In other words, while the phase of the lowest harmonics is hardly affected by molecular alignment, the harmonics above $\mathrm{H} 23$ present an important phase jump when the molecules are aligned. Similar phase jumps were recently measured by other techniques [4-6]. 
Phase jumps in the harmonic emission can be induced by at least two mechanisms: two-centre interference related to the structure of a given molecular orbital [16], or interference between contributions from several molecular orbitals [8]. A simple way to distinguish them is to study the position of the phase jump as a function of the laser intensity: a structural phase jump should be fixed while a multiple-orbital jump will move [8]. We have repeated our experiment at a lower generating intensity $\left(1 \times 10^{14} \mathrm{~W} \mathrm{~cm}^{-2}\right)$. The sudden increase in the diffracted light occurs at a lower harmonic order $(\mathrm{H} 19$, see figure 6): the position of the phase jump varies with the laser intensity. This experiment thus confirms the important role of multiple orbitals in high harmonic generation from $\mathrm{CO}_{2}$ molecules.

\section{Conclusion}

In conclusion, we have shown that high order harmonic transient gratings can be used to perform high-contrast phasesensitive pump-probe measurements. We have used this technique to measure the phase of the harmonic emission from aligned molecules, showing that the phase modulation depth decreases with harmonic order in $\mathrm{N}_{2}$. In $\mathrm{CO}_{2}$ we have observed strong phase jumps whose exact magnitude cannot be extracted with this measurement because of phase matching issues. Still, we have been able to study the intensity dependence of these phase jumps, which is an important information for the understanding of the generating mechanism from multiple molecular orbitals. Extracting the phase modulation from the diffraction pattern requires that the grating has a well-known profile. In the case of one or two-photon molecular excitation this condition is naturally fulfilled, which make this technique important for the future of dynamic imaging of molecules with high order harmonics.

\section{References}

[1] Itatani J, Levesque J, Zeidler D, Niikura H, Pepin H, Kieffer J C, Corkum P B and Villeneuve D M 2004 Nature 432867

[2] Marangos J P, Baker S, Kajumba N, Robinson J S, Tisch J W G and Torres R 2008 Phys. Chem. Chem. Phys. 1035

[3] Levesque J, Mairesse Y, Dudovich N, P'epin H, Kieffer J C, Corkum P B and Villeneuve D M 2007 Phys. Rev. Lett. 99243001

[4] Wagner N, Zhou X, Lock R, Li W, Wüest A, Murnane M and Kapteyn H 2007 Phys. Rev. A 76061403

[5] Kanai T, Takahashi E J, Nabekawa Y and Midorikawa K 2008 Phys. Rev. A 77041402

[6] Boutu W et al 2008 Nat. Phys. 4545

[7] Zhou X et al 2008 Phys. Rev. Lett. 100073902

[8] Smirnova O et al 2009 Nature 460972

[9] Levenson M D 1982 Introduction to Nonlinear Laser Spectroscopy (New York: Academic)

[10] Mairesse Y, Zeidler D, Dudovich N, Levesque J, Villeneuve D M and Corkum P B 2008 Phys. Rev. Lett. 100143903

[11] Rosca-Pruna F and Vrakking M J J 2001 Phys. Rev. Lett. 87153902

[12] Stapelfeldt H and Seideman T 2003 Rev. Mod. Phys. 75543

[13] Mairesse Y et al 2007 Opt. Lett. 32436

[14] Dooley P W et al 2003 Phys. Rev. A 68023406

[15] Salières P, L'Huillier A and Lewenstein M 1995 Phys. Rev. Lett. 743776

[16] Lein M, Hay N, Velotta R, Marangos J P and Knight P L 2002 Phys. Rev. A 66023805

[17] Mairesse Y, Levesque J, Dudovich N, Corkum P B and Villeneuve D M 2008 J. Mod. Opt. 552591

[18] Wabnitz H et al 2006 Eur. Phys. J. D 40305

[19] Haessler S et al 2010 Nat. Phys. in press 